\title{
The "Golden Age" of Probiotics: A Systematic Review and Meta-Analysis of Randomized and Observational Studies in Preterm Infants
}

\author{
Elda Dermyshi ${ }^{a, b}$ Yizhong Wang ${ }^{a}$ Chongbin Yan $^{b}$ Wenchao Hong ${ }^{b}$ \\ Gang Qiu $^{\mathrm{b}}$ Xiaohui Gong ${ }^{\mathrm{b}}$ Ting Zhang $^{\mathrm{a}}$ \\ Departments of a Gastroenterology, Hepatology, and Nutrition, and ' Neonatology, Shanghai Children's Hospital, \\ Shanghai Jiao Tong University, Shanghai, PR China
}

\section{Keywords}

Probiotics · Preterm - Very low birth weight infants .

Necrotizing enterocolitis · Mortality $\cdot$ Sepsis

\begin{abstract}
Background: Over the last few years, probiotics have been one of the most studied interventions in neonatal medicine. Objectives: The aim of this work was to analyse all studies (randomized controlled trials, RCTs, and observational studies) assessing the use of probiotics in very low birth weight (VLBW) preterm infants. Search Methods: A systematic literature search was conducted using PubMed, Embase, Cochrane Library, and Web of Science. The data from RCTs and observational studies were pooled and analysed separately. Selection Criteria: RCTs and observational studies that enrolled VLBW infants with enteral administration of probiotics were considered. Extracted study data included probiotic characteristics and at least 1 clinical outcome (necrotizing enterocolitis [NEC], late-onset sepsis or all-cause mortality). Data Collection and Analysis: Forty-four studies were eligible for our review: 30 RCTs and 14 observational studies. Severe NEC rates (stage II or more) and all-cause mortality were reduced among the probiotic groups in both the RCTs
\end{abstract} Elda Dermyshi and Yizhong Wang contributed equally to this study.

\section{KARGER}

(c) 2017 S. Karger AG, Basel

E-Mail karger@karger.com

www.karger.com/neo
(RR 0.57, 95\% Cl 0.47-0.70, and RR 0.77, 95\% Cl 0.65-0.92, respectively) and the observational studies (RR $0.51,95 \% \mathrm{Cl}$ $0.37-0.70$, and RR $0.71,95 \% \mathrm{Cl} 0.62-0.81$, respectively). Furthermore, there was a $12 \%$ reduction in the risk of sepsis in RCTs and a 19\% reduction in observational studies. The meta-analysis of observational studies showed a reduction in the risk of NEC in extremely low birth weight infants. However, this was not statistically significant. Conclusions: This meta-analysis of RCT and observational studies found that the use of probiotics was beneficial for the prevention of severe NEC, late-onset sepsis, and all-cause mortality in VLBW infants.

(c) 2017 S. Karger AG, Basel

\section{Background}

Necrotizing enterocolitis (NEC) and sepsis are increasingly important contributors to mortality because of more preterm infants surviving the first week of life [1]. NEC is the most common serious acquired disease of the gastrointestinal tract in preterm infants [2]. It is characterized by ischemic necrosis of the intestinal mucosa,

Elda Dermyshi, MD

Department of Neonatology, Shanghai Children's Hospital

Shanghai Jiao Tong University

No. 355, Luding Road, Shanghai 200062 (PR China)

E-Mail elda.dermyshi@yahoo.com

Ting Zhang, MD, PhD

Department of Gastroenterology, Hepatology, and Nutrition, Shanghai Children's Hospital, Shanghai Jiao Tong University, No. 355, Luding Road, Shanghai 200062 (PR China)

E-Mail zhangt@shchildren.com.cn 
with an excessive inflammatory process and invasion of enteric gas-forming organisms, and is a leading cause of morbidity and mortality among preterm infants, with the risk of developing NEC inversely proportional to birth weight [3]. The aetiology of NEC has not been fully explained, but it seems to be multifactorial, involving the immaturity of intestinal host defences and abnormal bacterial colonization $[4,5]$. In vitro evidence showed that pathogenic flora attached to the epithelial cells of preterm infants much more easily than to those of term infants, and some studies indicated that commensal bacteria could inhibit or reduce inflammatory signalling in intestinal epithelial cells [6]. In contrast to term infants, the microbiome of premature infants has a smaller proportion of beneficial bacteria and higher numbers of pathogenic bacteria likely owing to frequent antibiotic use, exposure to the hospital environment, and artificial feeding [7]. This may predispose them to a failure of postnatal evolution of critical innate defences and lead to NEC [8]. The hypothesis supporting the use of probiotic bacteria to prevent NEC and sepsis is that their administration to the preterm infant will encourage gut microbiota resembling that of the term infant, strengthen intestinal barrier function, and, thereby, protect the infant.

There is an increasing interest in probiotic intervention and evidence for the effectiveness of probiotics in preventing severe NEC, late-onset sepsis, and overall mortality in very low birth weight (VLBW) infants $[9,10]$. There is still insufficient clinical trial data available on which to consider the merits of both the safety and efficacy of providing probiotics to extremely low birth weight (ELBW) infants. To address these investigative questions in preterm infants, especially in ELBW infants, randomized controlled trials (RCTs) are not always easy or ethical to conduct. Instead, well-designed observational studies may be the next practicable method to address these types of questions as they have been shown to provide results similar to RCTs, challenging the belief that observational studies are second-rate $[11,12]$. They reflect routine practice, which allows for the evaluation of effectiveness and safety in large populations that include ELBW patients who are under-represented in, or completely excluded from RCTs. In addition, they are sufficiently large to allow the study of rare events and are readily available for analysis by researchers, without the time and monetary costs common to large RCTs.

We therefore aimed to conduct a systematic review on RCTs and observational studies, to investigate the effects of probiotics, and to compare the efficacy and safety of probiotic administration in the prevention of severe (stage II or more) NEC, late-onset sepsis, and mortality in VLBW infants. The secondary objective was to conduct a subgroup analysis to study the effect of probiotics in ELBW infants and to analyse the effect of different species or combinations of probiotics in VLBW infants.

\section{Materials and Methods}

We followed the PRISMA guidelines [13], MOOSE guidelines [14], and the Cochrane Handbook of Systematic Reviews of Interventions approach [15] for conducting and reporting systematic reviews and meta-analyses of RCTs and observational studies.

\section{Search Methods for the Identification of Studies}

The search was conducted on MEDLINE/PubMed, Embase, Cochrane Library, and Web of Science to identify RCTs and observational studies that addressed the effect of probiotics in VLBW infants. The databases were screened for publications from the earliest available date until July 30, 2016. Only articles written in English were considered. The keywords searched included combinations of "probiotics," "preterm," "necrotizing enterocolitis," "sepsis," "infant," and "very-low-birth-weight."

\section{Eligibility Criteria}

RCTs and observational studies involving VLBW $(<1,500 \mathrm{~g})$ preterm ( $<34$ weeks gestational age) infants with enteral administration of probiotics initiated within 10 days were included.

\section{Selection of Studies}

Paired reviewers (E.D., Y.W.) independently screened titles/ abstracts and then full texts for eligibility, assessed risk of bias, and collected data from each included study. Any disagreement between the 2 reviewers was resolved through discussion or adjudication by a third reviewer (C.Y.). In case of duplicate publications, only the most recent and updated report of the study was included.

\section{Risk of Bias and Quality of Evidence Assessment}

The Cochrane Risk-of-Bias Tool was used to assess the risk of bias of each RCTs [16]. The quality of the evidence of outcomes was rated by the Grading of Recommendations Assessment, Development, and Evaluation (GRADE) approach [17]. Furthermore, the quality of included observational trials was assessed using the Newcastle-Ottawa Scale (NOS) [18]: in which 0-3 stars indicate poor study quality, 4-6 stars indicate acceptable study quality, and 7-9 stars indicate good study quality.

\section{Data Extraction}

From each eligible study the following information was collected: study characteristics (e.g., author name, year of publication, country, sample size, patient characteristics, type of probiotic, duration of intervention, dosage) and at least 1 clinical outcome:

- Severe NEC (stage II or more) according to the modified Bell staging criteria

- Late-onset sepsis (confirmed with a positive blood culture)

- All-cause mortality 
Table 1. Characteristics of the included RCT studies

\begin{tabular}{|c|c|c|c|c|c|c|c|c|c|c|}
\hline $\begin{array}{l}\text { First author [Ref.], } \\
\text { year (country) }\end{array}$ & $\begin{array}{l}\text { Infants } \\
\text { on probi- } \\
\text { otics, } n\end{array}$ & $\begin{array}{l}\text { Control } \\
\text { group, } n\end{array}$ & $\begin{array}{l}\text { Inclusion } \\
\text { criteria } \\
(\mathrm{BW} / \mathrm{GA})\end{array}$ & $\begin{array}{l}\text { Strains, doses } \\
\text { and duration }\end{array}$ & $\begin{array}{l}\text { NEC } \\
\text { probiotics } \\
\text { (stage } \geq 2), n\end{array}$ & $\begin{array}{l}\text { NEC } \\
\text { controls } \\
(\text { stage } \geq 2), n\end{array}$ & $\begin{array}{l}\text { Sepsis pro- } \\
\text { biotics (culture } \\
\text { positive), } n\end{array}$ & $\begin{array}{l}\text { Sepsis controls } \\
\text { (culture } \\
\text { positive), } n\end{array}$ & $\begin{array}{l}\text { Mortality } \\
\text { probiotics } \\
\text { (all cause), } n\end{array}$ & $\begin{array}{l}\text { Mortality } \\
\text { controls } \\
\text { (all cause), } n\end{array}$ \\
\hline $\begin{array}{l}\text { Al-Hosni [19], } \\
2012 \text { (USA) }\end{array}$ & 50 & 51 & $<1,000 \mathrm{~g}$ & $\begin{array}{l}\text { L. rhamnosus GG and } B \text {. } \\
\text { infantis }\left(0.5 \times 10^{9} \mathrm{CFU} \text { each }\right. \\
\text { probiotic, } \mathrm{OD} \text { until } \\
34 \text { weeks) }\end{array}$ & $2 / 50$ & $2 / 51$ & $13 / 50$ & $16 / 51$ & $3 / 50$ & $3 / 50$ \\
\hline $\begin{array}{l}\text { Bin-Nun [20], } \\
2005 \text { (Israel) }\end{array}$ & 72 & 73 & $<1,500 \mathrm{~g}$ & $\begin{array}{l}\text { B. infantis, S. thermophilus, } B \text {. } \\
\text { bifidum }\left(0.35 \times 10^{9} \text { CFU each }\right. \\
\text { probiotic, OD until } 36 \text { weeks })\end{array}$ & $1 / 72$ & $10 / 73$ & $31 / 72$ & $24 / 73$ & $6 / 72$ & $17 / 73$ \\
\hline $\begin{array}{l}\text { Braga [21], } \\
2011 \text { (Brazil) }\end{array}$ & 119 & 112 & $750-1,499 \mathrm{~g}$ & $\begin{array}{l}\text { L. case } \text { and B. breve } \\
\left(3.5 \times 10^{7} \text { to } 3.5 \times 10^{9}\right. \\
\text { CFU, OD for } 28 \text { days })\end{array}$ & $0 / 119$ & $4 / 112$ & $40 / 119$ & $42 / 112$ & $26 / 119$ & $27 / 112$ \\
\hline $\begin{array}{l}\text { Costalos [62], } \\
2003 \text { (Greece) } \\
\end{array}$ & 51 & 36 & $28-32$ weeks & $\begin{array}{l}\text { S. boulardii } \\
\left(1 \times 10^{9} \mathrm{CFU}, \mathrm{BD} \text { for } 30 \text { days }\right)\end{array}$ & $5 / 51$ & $6 / 36$ & $3 / 51$ & $3 / 36$ & NR & NR \\
\hline $\begin{array}{l}\text { Costeloe [22], } \\
2016 \text { (UK) }\end{array}$ & 650 & 660 & $23-30$ weeks & $\begin{array}{l}\text { B. breve }(\text { BBG 001) } \\
\left(1.6 \times 10^{9} \mathrm{CFU} / \text { day until } 36\right. \\
\text { weeks })\end{array}$ & $61 / 650$ & $66 / 660$ & $73 / 650$ & $77 / 660$ & $54 / 650$ & $56 / 660$ \\
\hline $\begin{array}{l}\text { Costeloe [22], } \\
2016 \text { (UK) }\end{array}$ & 317 & 327 & $<1,000 \mathrm{~g}$ & $\begin{array}{l}\text { B. breve }(\mathrm{BBG} 001) \\
\left(1.6 \times 10^{9} \mathrm{CFU} / \text { day until } 36\right. \\
\text { weeks })\end{array}$ & $50 / 317$ & $53 / 327$ & $63 / 317$ & $61 / 327$ & $46 / 317$ & $55 / 327$ \\
\hline $\begin{array}{l}\text { Dani [23], } \\
2002 \text { (Italy) } \\
\end{array}$ & 295 & 290 & $\begin{array}{l}<33 \text { weeks } \\
\text { or }<1,500 \mathrm{~g}\end{array}$ & $\begin{array}{l}\text { Lactobacillus GG }\left(6.0 \times 10^{9}\right. \\
\text { CFU/day until discharge })\end{array}$ & $4 / 295$ & $8 / 290$ & $14 / 295$ & $12 / 290$ & $0 / 295$ & $2 / 290$ \\
\hline $\begin{array}{l}\text { Demirel [24], } \\
2013 \text { (Turkey) }\end{array}$ & 135 & 136 & $\begin{array}{l}\leq 32 \text { weeks } \\
\text { and } \leq 1,500 \mathrm{~g}\end{array}$ & $\begin{array}{l}\text { S. boulardii } \\
\left(5 \times 10^{9} \mathrm{CFU}, \mathrm{BD} \text { until }\right. \\
\text { discharge })\end{array}$ & $6 / 135$ & $7 / 136$ & $20 / 135$ & $21 / 136$ & $5 / 135$ & $5 / 136$ \\
\hline $\begin{array}{l}\text { Dilli [25], } \\
2015\end{array}$ & 100 & 100 & $\begin{array}{l}<32 \text { weeks } \\
\text { and }<1,500 \mathrm{~g}\end{array}$ & $\begin{array}{l}\text { B. lactis }\left(5 \times 10^{9} \mathrm{CFU}\right. \\
\text { for } 8 \text { weeks })\end{array}$ & $2 / 100$ & $18 / 100$ & $8 / 100$ & $13 / 100$ & $3 / 100$ & $12 / 100$ \\
\hline $\begin{array}{l}\text { Dutta [26], } \\
2015 \text { (India) }\end{array}$ & 38 & 35 & $27-33$ weeks & $\begin{array}{l}\text { L. acidophilus, } L . \text { rhamnosus, } \\
\text { B. longum, and } S . \text { boulardii } \\
\left(1 \times 10^{10} \text { CFU, BD for } 21 \text { days }\right)\end{array}$ & $1 / 38$ & $0 / 35$ & $3 / 38$ & $6 / 35$ & $3 / 38$ & $2 / 35$ \\
\hline $\begin{array}{l}\text { Fernández- } \\
\text { Carrocera [27], } \\
2013 \text { (Mexico) }\end{array}$ & 75 & 75 & $<1,500 \mathrm{~g}$ & $\begin{array}{l}\text { L. acidophilus } 1 \times 10^{8} \\
\text { CFU/g, L. rhamnosus } \\
4.4 \times 10^{8} \mathrm{CFU} / \mathrm{g}, \text { L. casei } \\
1 \times 10^{9} \mathrm{CFU} / \mathrm{g}, \text { L. plantarum } \\
1.76 \times 10^{8} \mathrm{CFU} / \mathrm{g} \\
\text { B. infantis } 2.76 \times 10^{7} \mathrm{CFU} / \mathrm{g} \text {, } \\
\text { S. thermophilus } 6.6 \times 10^{5} \\
\text { CFU/g }\end{array}$ & $6 / 75$ & $12 / 75$ & NR & NR & $1 / 75$ & $7 / 75$ \\
\hline $\begin{array}{l}\text { Fuji [28], } \\
2006 \text { (Japan) }\end{array}$ & 11 & 8 & $<34$ weeks & $\begin{array}{l}\text { B. breve }(\mathrm{M}-16 \mathrm{~V}) 1 \times 10^{9} \mathrm{CFU} \text {, } \\
\text { BD until discharge) }\end{array}$ & $0 / 11$ & $0 / 8$ & $1 / 11$ & $1 / 8$ & NR & NR \\
\hline $\begin{array}{l}\text { Hays [29], } \\
2015 \text { (France) }\end{array}$ & 50 & 52 & $\begin{array}{l}700-1,600 \mathrm{~g} \\
\text { and } 25-31 \\
\text { weeks }\end{array}$ & $\begin{array}{l}\text { B. lactis }\left(1 \times 10^{9} \mathrm{CFU} / \text { day }\right. \\
\text { for } 4-6 \text { weeks })\end{array}$ & $2 / 50$ & $3 / 52$ & $9 / 50$ & $10 / 52$ & $1 / 50$ & $1 / 52$ \\
\hline $\begin{array}{l}\text { Hays [29], } \\
2015 \text { (France) }\end{array}$ & 48 & 52 & $\begin{array}{l}700-1,600 \mathrm{~g} \\
\text { and } 25-31 \\
\text { weeks }\end{array}$ & $\begin{array}{l}\text { B. longum }\left(1 \times 10^{9}\right. \\
\text { CFU/day for } 4-6 \text { weeks })\end{array}$ & $1 / 48$ & $3 / 52$ & $8 / 48$ & $10 / 52$ & $1 / 48$ & $1 / 52$ \\
\hline $\begin{array}{l}\text { Hays [29], } \\
2015 \text { (France) }\end{array}$ & 47 & 52 & $\begin{array}{l}700-1,600 \mathrm{~g} \\
\text { and } 25-31 \\
\text { weeks }\end{array}$ & $\begin{array}{l}\text { B. lactis and B. longum } \\
\left(1 \times 10^{9} \mathrm{CFU} / \text { day for }\right. \\
4-6 \text { weeks })\end{array}$ & $5 / 47$ & $3 / 52$ & $8 / 47$ & $10 / 52$ & $1 / 47$ & $1 / 52$ \\
\hline $\begin{array}{l}\text { Jacobs } \\
\text { (ProPrem) [30], } \\
2013 \text { (Australia) } \\
\end{array}$ & 548 & 551 & $\begin{array}{l}<32 \text { weeks } \\
\text { and }<1,500 \mathrm{~g}\end{array}$ & $\begin{array}{l}\text { B. infantis, S. thermophilus, } \\
\text { and B. lactis }\left(1 \times 10^{9} \mathrm{CFU} /\right. \\
\text { day until discharge })\end{array}$ & $11 / 548$ & $24 / 551$ & $72 / 548$ & $89 / 551$ & $27 / 548$ & $28 / 551$ \\
\hline $\begin{array}{l}\text { Jacobs } \\
\text { (ProPrem), [30] } \\
2013 \text { (Australia) } \\
\text { subgroup }\end{array}$ & 232 & 239 & $<1,000 \mathrm{~g}$ & $\begin{array}{l}\text { B. infantis, S. thermophilus, } \\
\text { and B. lactis }\left(1 \times 10^{9} \mathrm{CFU} /\right. \\
\text { day until discharge })\end{array}$ & $10 / 232$ & $14 / 239$ & $53 / 232$ & $58 / 239$ & NR & NR \\
\hline $\begin{array}{l}\text { Kanic [31], } \\
2015 \text { (Slovenia) }\end{array}$ & 40 & 40 & $\begin{array}{l}<33 \text { weeks } \\
\text { and }<1,500 \mathrm{~g}\end{array}$ & $\begin{array}{l}\text { L. acidophilus, E. faecium, } \\
\text { and } B \text {. infantis }\left(0.6 \times 10^{7} \mathrm{CFU} \text {, }\right. \\
\text { BD until discharge })\end{array}$ & $0 / 40$ & $5 / 40$ & $16 / 40$ & $29 / 40$ & $2 / 40$ & $3 / 40$ \\
\hline $\begin{array}{l}\text { Lin [32], } 2005 \\
\text { (Taiwan) }\end{array}$ & 180 & 187 & $<1,500 \mathrm{~g}$ & $\begin{array}{l}\text { L. acidophilus and } B \text {. } \\
\text { infantis (each } 2 \times 10^{9} \mathrm{CFU} \\
\text { until discharge) }\end{array}$ & $2 / 180$ & $10 / 187$ & $22 / 180$ & $36 / 187$ & $7 / 180$ & $20 / 187$ \\
\hline $\begin{array}{l}\text { Lin [33], } \\
2008 \text { (Taiwan) }\end{array}$ & 217 & 217 & $<1,500 \mathrm{~g}$ & $\begin{array}{l}\text { B. bifidum and L. acidophilus } \\
\text { (each } 1 \times 10^{9} \mathrm{CFU}, \mathrm{BD} \text { for } \\
6 \text { weeks) }\end{array}$ & $4 / 217$ & $14 / 217$ & $40 / 217$ & $24 / 217$ & $2 / 217$ & $9 / 217$ \\
\hline $\begin{array}{l}\text { Manzoni [34], } \\
2006 \text { (Italy) } \\
\end{array}$ & 39 & 41 & $<1,500 \mathrm{~g}$ & $\begin{array}{l}\text { Lactobacillus GG casei subspe- } \\
\text { cies rhamnosus }\left(6 \times 10^{9} / \mathrm{kg}\right)\end{array}$ & $1 / 39$ & $3 / 41$ & $19 / 39$ & $22 / 41$ & $5 / 39$ & $6 / 41$ \\
\hline $\begin{array}{l}\text { Manzoni [35], } \\
2009 \text { (Italy) }\end{array}$ & 151 & 168 & $<1,500 \mathrm{~g}$ & $\begin{array}{l}\text { Lactobacillus GG subspecies } \\
\text { rhamnosus }\left(6 \times 10^{9} \mathrm{CFU} /\right. \\
\text { day for } 4 \text { weeks })\end{array}$ & $0 / 151$ & $3 / 168$ & $7 / 151$ & $9 / 168$ & $6 / 151$ & $4 / 168$ \\
\hline $\begin{array}{l}\text { Manzoni [36], } \\
2014 \text { (Italy) } \\
\end{array}$ & 238 & 247 & $<1,500 \mathrm{~g}$ & $\begin{array}{l}\text { Lactobacillus GG } \\
\left(6 \times 10^{9} \mathrm{CFU} / \text { day }\right)\end{array}$ & $0 / 238$ & $5 / 247$ & NR & NR & $9 / 238$ & $5 / 247$ \\
\hline
\end{tabular}


Table 1 (continued)

\begin{tabular}{|c|c|c|c|c|c|c|c|c|c|c|}
\hline $\begin{array}{l}\text { First author [Ref.], } \\
\text { year (country) }\end{array}$ & $\begin{array}{l}\text { Infants } \\
\text { on probi- } \\
\text { otics, } n\end{array}$ & $\begin{array}{l}\text { Control } \\
\text { group, } n\end{array}$ & $\begin{array}{l}\text { Inclusion } \\
\text { criteria } \\
(\mathrm{BW} / \mathrm{GA})\end{array}$ & $\begin{array}{l}\text { Strains, doses } \\
\text { and duration }\end{array}$ & $\begin{array}{l}\text { NEC } \\
\text { probiotics } \\
(\text { stage } \geq 2), n\end{array}$ & $\begin{array}{l}\text { NEC } \\
\text { controls } \\
(\text { stage } \geq 2), n\end{array}$ & $\begin{array}{l}\text { Sepsis pro- } \\
\text { biotics (culture } \\
\text { positive), } n\end{array}$ & $\begin{array}{l}\text { Sepsis controls } \\
\text { (culture } \\
\text { positive), } n\end{array}$ & $\begin{array}{l}\text { Mortality } \\
\text { probiotics } \\
\text { (all cause), } n\end{array}$ & $\begin{array}{l}\text { Mortality } \\
\text { controls } \\
\text { (all cause), } n\end{array}$ \\
\hline $\begin{array}{l}\text { Mihatsch [37], } \\
2010 \text { (Germany) }\end{array}$ & 91 & 89 & $\begin{array}{l}<30 \text { weeks } \\
\text { and }<1,500 \mathrm{~g}\end{array}$ & $\begin{array}{l}\text { B. lactis }\left(2 \times 10^{9} \mathrm{CFU} / \mathrm{kg} /\right. \\
\text { day for } 6 \text { weeks })\end{array}$ & $2 / 91$ & $4 / 89$ & $28 / 91$ & $29 / 89$ & $2 / 91$ & $1 / 89$ \\
\hline $\begin{array}{l}\text { Oncel [38], } \\
2014 \text { (Turkey) }\end{array}$ & 200 & 200 & $\begin{array}{l}<32 \text { weeks } \\
\text { and } \leq 1,500 \mathrm{~g}\end{array}$ & $\begin{array}{l}\text { L. reuteri }\left(1 \times 10^{8} \mathrm{CFU} \text {, }\right. \\
\text { OD until discharge })\end{array}$ & $8 / 200$ & $10 / 200$ & $13 / 200$ & $25 / 200$ & $15 / 200$ & $20 / 200$ \\
\hline $\begin{array}{l}\text { Patole [39], } \\
2014 \text { (Australia) }\end{array}$ & 77 & 76 & $\begin{array}{l}<33 \text { weeks } \\
\text { and }<1,500 \mathrm{~g}\end{array}$ & $\begin{array}{l}\text { B. breve M16-V HM } \\
\left(3 \times 10^{9} \text { CFU OD; } 1.5 \times 10^{9}\right. \\
\text { CFU OD for newborns } \\
\leq 27 \text { weeks until they reached } \\
50 \mathrm{~mL} / \mathrm{kg} / \text { day enteral feeds })\end{array}$ & $0 / 77$ & $1 / 76$ & $17 / 77$ & $12 / 76$ & $0 / 77$ & $0 / 76$ \\
\hline $\begin{array}{l}\text { Rojas [40], } \\
2012 \text { (Colombia) }\end{array}$ & 176 & 184 & $\leq 1,500 \mathrm{~g}$ & $\begin{array}{l}\text { L. reuteri }\left(1 \times 10^{8} \mathrm{CFU}\right. \\
\text { until discharge })\end{array}$ & $6 / 176$ & $10 / 184$ & $28 / 176$ & $33 / 184$ & NR & NR \\
\hline $\begin{array}{l}\text { Rougé [41], } \\
2009 \text { (France) }\end{array}$ & 45 & 49 & $<1,500 \mathrm{~g}$ & $\begin{array}{l}\text { B. longum, and L. rhamnosus } \\
\left(1 \times 10^{8} \mathrm{CFU} / \text { day until }\right. \\
\text { discharge })\end{array}$ & $2 / 45$ & $1 / 49$ & $15 / 45$ & $13 / 49$ & $2 / 45$ & $4 / 49$ \\
\hline $\begin{array}{l}\text { Saengtawesin [42], } \\
2014 \text { (Thailand) }\end{array}$ & 31 & 29 & $\begin{array}{l}\leq 34 \text { weeks } \\
\text { and } \leq 1,500 \mathrm{~g}\end{array}$ & $\begin{array}{l}\text { L. acidophilus, B. bifidum } \\
\left(1 \times 10^{9} \mathrm{CFU} \text { each, }\right. \\
\text { total } 125 \mathrm{mg} / \mathrm{kg}, \mathrm{BD} \text { until } \\
\text { discharge })\end{array}$ & $1 / 31$ & $1 / 29$ & $2 / 31$ & $1 / 29$ & $0 / 31$ & $0 / 29$ \\
\hline $\begin{array}{l}\text { Samanta [43], } \\
2009 \text { (India) }\end{array}$ & 91 & 95 & $\begin{array}{l}<32 \text { weeks } \\
\text { and }<1,500 \mathrm{~g}\end{array}$ & $\begin{array}{l}\text { B. infantis, B. bifidum, } \\
\text { B. longum, and L. acidophilus } \\
\text { (each } 2.5 \times 10^{9} \mathrm{CFU}, \mathrm{BD} \\
\text { until discharge) }\end{array}$ & $5 / 91$ & $15 / 95$ & $13 / 91$ & $28 / 95$ & $4 / 91$ & $14 / 95$ \\
\hline $\begin{array}{l}\text { Sari [44], } \\
2011 \text { (Turkey) }\end{array}$ & 110 & 111 & $\begin{array}{l}<33 \text { weeks } \\
\text { or }<1,500 \mathrm{~g}\end{array}$ & $\begin{array}{l}\text { L. sporogenes }(0.35 \times \\
\left.10^{9} \text { CFU, OD; NR }\right)\end{array}$ & $6 / 110$ & $10 / 111$ & $29 / 110$ & $26 / 111$ & $3 / 110$ & $3 / 111$ \\
\hline $\begin{array}{l}\text { Serce [45], } \\
2013 \text { (Turkey) }\end{array}$ & 104 & 104 & $\begin{array}{l}\leq 32 \text { weeks } \\
\text { and } \leq 1,500 \mathrm{~g}\end{array}$ & $\begin{array}{l}\text { S. boulardii }\left(0.5 \times 10^{9} \mathrm{CFU} /\right. \\
\mathrm{kg} \text { per } \\
\text { dose, } \mathrm{BD})\end{array}$ & $7 / 104$ & $7 / 104$ & $19 / 104$ & $25 / 104$ & $5 / 104$ & $4 / 104$ \\
\hline $\begin{array}{l}\text { Tewari [46], } \\
2015 \text { (India) }\end{array}$ & 123 & 121 & $<34$ weeks & $\begin{array}{l}\text { B. clausii }\left(8 \times 10^{8} \mathrm{CFU}, \mathrm{TD}\right. \\
\text { for } 3-5 \text { weeks })\end{array}$ & $0 / 123$ & $0 / 121$ & $20 / 123$ & $25 / 121$ & $12 / 123$ & $14 / 121$ \\
\hline $\begin{array}{l}\text { Totsu [47], } \\
2014 \text { (Japan) }\end{array}$ & 153 & 130 & $<1,500 \mathrm{~g}$ & $\begin{array}{l}\text { B. bifidum }\left(2.5 \times 10^{9}\right. \\
\text { CFU, divided in } 2 \text { doses } \\
\text { until }>2 \mathrm{~kg})\end{array}$ & $0 / 153$ & $0 / 130$ & $6 / 153$ & $10 / 130$ & $2 / 153$ & $0 / 130$ \\
\hline
\end{tabular}

CFU, colony-forming unit; BW, birth weight; GA, gestational age; NR, not reported.

Statistical Analysis

All the statistical analyses were performed using the Stata 12.0 (Stata Corporation, College Station, TX, USA) and RevMan 5.3 software (The Nordic Cochrane Centre, The Cochrane Collaboration). RCTs and observational studies were analysed separately. For each trial, relative risk (RR) with a 95\% confidence interval (95\% CI) for NEC, sepsis, and mortality was calculated using the Mantel-Haenszel method. In the event of zero events in both groups, the RR was not estimable. Both the fixed-effects model and random-effects model were considered, depending on the heterogeneity of the included studies. The heterogeneity of the studies was assessed using $I^{2}$. A value of $I^{2}<50 \%$ indicated homogeny and the fixed-effects model was used in the analysis. In contrast, $I^{2}$ $>50 \%$ was interpreted as representing significant heterogeneity and the random-effects model was used.

There was considerable clinical heterogeneity in probiotics formulations and treatment duration in the observational studies so data were analysed using a random-effects model. The publication bias in this meta-analysis was first visually evaluated by funnel plots and further evaluated using the Egger and Begg tests. A subgroup analysis was performed to investigate the effect of probiotics in ELBW infants and the effect of different species of probiotics in VLBW.

\section{Results}

\section{Description of Studies}

A total of 1,032 articles were identified by the initial databases search (online suppl. Fig. 1; for all online suppl. material, see www.karger.com/doi/10.1159/000454668). A total of 585 articles were excluded as duplicates or for language, meta-analysis or other reasons. Thus, 72 were potentially eligible after title and abstract screening, and 44 studies met our inclusion criteria. Thirty RCTs with 8,622 patients and 14 observational studies with 13,779 patients were included. Regarding the enrolment criteria, the included trials were highly variable in birth weight, gestational age, dose, timing, and type of probiotics. Twenty-nine included RCT studies reported on severe stage II-III NEC outcome incorporating 4,304 infants treated with probiotics and 4,231 control infants. Twenty-eight included RCT studies reported on late-onset sepsis outcome involving 4,042 infants treated with probiotics and 3,945 control infants. Twenty-seven in- 
Table 2. Characteristics of the included observational studies

\begin{tabular}{|c|c|c|c|c|c|c|c|c|c|c|}
\hline $\begin{array}{l}\text { First author [Ref.], } \\
\text { year (country) }\end{array}$ & $\begin{array}{l}\text { Infants } \\
\text { on probi- } \\
\text { otics, } n\end{array}$ & $\begin{array}{l}\text { Control } \\
\text { group, } n\end{array}$ & $\begin{array}{l}\text { Inclusion } \\
\text { criteria } \\
(\mathrm{BW} / \mathrm{GA})\end{array}$ & Probiotic used (doses) & $\begin{array}{l}\text { NEC } \\
\text { Probiotics, } \\
n\end{array}$ & $\begin{array}{l}\text { NEC } \\
\text { controls, } \\
n\end{array}$ & $\begin{array}{l}\text { Sepsis pro- } \\
\text { biotics (culture } \\
\text { positive), } n\end{array}$ & $\begin{array}{l}\text { Sepsis con- } \\
\text { trols (culture } \\
\text { positive), } n\end{array}$ & $\begin{array}{l}\text { Mortality } \\
\text { probiotics } \\
\text { (all cause), } n\end{array}$ & $\begin{array}{l}\text { Mortality } \\
\text { controls } \\
\text { (all cause), } n\end{array}$ \\
\hline $\begin{array}{l}\text { Bonsante [48], } \\
2013 \text { (France) }\end{array}$ & 347 & 783 & $\begin{array}{l}>24 \text { and } \\
<31 \text { weeks }\end{array}$ & $\begin{array}{l}\text { L. rhamnosus }(2 \times \\
\left.10^{8} \mathrm{CFU} \text { BD until } 36 \text { weeks }\right)\end{array}$ & $4 / 347$ & $42 / 783$ & $37 / 347$ & $130 / 783$ & $8 / 347$ & $38 / 783$ \\
\hline $\begin{array}{l}\text { Dang [49], } \\
2015 \text { (USA) }\end{array}$ & 128 & 135 & $\begin{array}{l}<1,250 \mathrm{~g} \text { and } \\
<28 \text { weeks }\end{array}$ & $\begin{array}{l}\text { L. rhamnosus GG/B. infantis } \\
\left(1 \times 10^{9} \mathrm{CFU} / \text { day until } 34 \text { week }\right)\end{array}$ & $2 / 128$ & $8 / 135$ & NR & NR & $19 / 128$ & $21 / 135$ \\
\hline $\begin{array}{l}\text { Guthmann [50], } \\
2015 \\
\text { (Switzerland) }\end{array}$ & 591 & 633 & $\begin{array}{l}400-1,500 \mathrm{~g} \\
\text { and }<32 \text { weeks }\end{array}$ & $\begin{array}{l}\text { L. acidophilus/B. infantis } \\
\text { (each } 1 \times 10^{9} \mathrm{CFU} / \text { day for } \\
10-14 \text { days) }\end{array}$ & $8 / 591$ & $33 / 633$ & NR & NR & $21 / 591$ & $32 / 633$ \\
\hline $\begin{array}{l}\text { Härtel [53], } \\
2014 \text { (Germany) }\end{array}$ & 3,789 & 1,562 & $\begin{array}{l}>22+6 \text { and } \\
32 \text { weeks or } \\
<1,500 \mathrm{~g}\end{array}$ & $\begin{array}{l}\text { L. acidophilus/B. infantis } \\
\left(1 \times 10^{9} \mathrm{CFU} / \text { day for } 14 \text { days }\right)\end{array}$ & $67 / 2,566$ & $44 / 1,043$ & $298 / 2,566$ & $115 / 1,043$ & $190 / 2,566$ & $108 / 1,043$ \\
\hline $\begin{array}{l}\text { Hoyos [51], } \\
1999 \text { (Colombia) }\end{array}$ & 102 & 103 & $<1,500 \mathrm{~g}$ & $\begin{array}{l}\text { L. acidophilus/B. infantis } \\
\left(5 \times 10^{8} / \text { day; NR }\right)\end{array}$ & $10 / 102$ & $26 / 103$ & $24 / 102$ & $23 / 103$ & $5 / 102$ & $17 / 103$ \\
\hline $\begin{array}{l}\text { Hunter [52], } \\
2012 \text { (USA) }\end{array}$ & 79 & 232 & $<1,000 \mathrm{~g}$ & $\begin{array}{l}\text { L. reuteri }\left(5.5 \times 10^{7} \mathrm{CFU} / \text { day }\right. \\
\text { until } 40 \text { weeks })\end{array}$ & $2 / 79$ & $35 / 232$ & $19 / 79$ & $72 / 232$ & NR & NR \\
\hline $\begin{array}{l}\text { Janvier [54], } \\
2014 \text { (Canada) }\end{array}$ & 294 & 317 & $<32$ weeks & $\begin{array}{l}\text { Mixture of Bifidobacterium } \\
\text { and Lactobacillus }\left(2 \times 10^{9} \mathrm{CFU} /\right. \\
\text { day until } 34 \text { weeks })\end{array}$ & $16 / 294$ & $31 / 317$ & $54 / 294$ & $57 / 317$ & $20 / 294$ & $31 / 317$ \\
\hline $\begin{array}{l}\text { Lambæk [55], } \\
2016 \text { (Denmark) }\end{array}$ & 333 & 381 & $<30$ weeks & $\begin{array}{l}\text { B. lactis } \mathrm{BB} 12 / \text { L. rhamnosus GG } \\
\left(1 \times 10^{8} \text { and } 1 \times 10^{9} \mathrm{CFU}\right)\end{array}$ & $23 / 333$ & $34 / 381$ & NR & NR & $54 / 333$ & $66 / 381$ \\
\hline $\begin{array}{l}\mathrm{Li}[56], \\
2013 \text { (California) } \\
\end{array}$ & 291 & 289 & $<1500 \mathrm{~g}$ & $\begin{array}{l}\text { Mixture of Streptococcus } \\
\text { and Bifidobacterium (NR) }\end{array}$ & $7 / 291$ & $8 / 289$ & NR & NR & $4 / 291$ & $3 / 289$ \\
\hline $\begin{array}{l}\text { Luoto [57], } \\
2010 \text { (Finland) }\end{array}$ & 418 & 1.9 & $\begin{array}{l}<30 \text { weeks } \\
\text { or }<1,500 \mathrm{~g}\end{array}$ & $\begin{array}{l}\text { Lactobacillus } \mathrm{GG}\left(6 \times 10^{9} \mathrm{CFU} /\right. \\
\text { day until discharge })\end{array}$ & $19 / 418$ & $61 / 1,900$ & NR & NR & NR & NR \\
\hline $\begin{array}{l}\text { Patole [58], } \\
2016 \text { (Australia) }\end{array}$ & 920 & 835 & $<34$ weeks & $\begin{array}{l}\text { B. breve M-16V }\left(1.5 \times 10^{9} \mathrm{CFU} /\right. \\
\left.\text { day and then } 3 \times 10^{9} \mathrm{CFU} / \text { day }\right)\end{array}$ & $12 / 920$ & $25 / 835$ & $82 / 920$ & $120 / 835$ & $37 / 920$ & $56 / 835$ \\
\hline $\begin{array}{l}\text { Repa [59], } \\
2015 \text { (Austria) }\end{array}$ & 230 & 233 & $<34$ weeks & $\begin{array}{l}\text { L. acidophilus/B. infantis } \\
\left(2 \times 10^{9} \mathrm{BD} ; \mathrm{NR}\right)\end{array}$ & $16 / 230$ & $24 / 233$ & $60 / 230$ & $78 / 233$ & $16 / 230$ & $30 / 233$ \\
\hline $\begin{array}{l}\text { Yamashiro [60], } \\
2010 \text { (Japan) }\end{array}$ & 338 & 226 & $<1,500 \mathrm{~g}$ & B. breve $\left(1 \times 10^{9} /\right.$ day $(\mathrm{NR})$ & $0 / 338$ & $6 / 226$ & $70 / 338$ & $65 / 226$ & $39 / 338$ & $38 / 226$ \\
\hline $\begin{array}{l}\text { Zampieri [61], } \\
2013 \text { (Japan) }\end{array}$ & 18 & 14 & $<1,500 \mathrm{~g}$ & $\begin{array}{l}\text { L. paracasei subspecies paracasei } \\
\text { F19 }\end{array}$ & $3 / 18$ & $6 / 14$ & NR & NR & NR & NR \\
\hline
\end{tabular}

CFU, colony-forming unit; BW, birth weight; GA, gestational age; NR, not reported.

cluded RCT studies reported on severe mortality outcome in 4,117 infants treated with probiotics and 4,039 control infants. There were 14 observational studies including 13,779 patients. The main characteristics of the included RCTs and observational studies are described in Tables 1 and 2. The characteristics of the excluded studies are summarized in online supplementary Table 1 . The study quality assessments of the trials according to the Cochrane risk-of-bias tool for RCTs and to the NOS for observational studies are summarized in online supplementary Tables 2 and 3. The evaluations of the level of evidence of outcomes according to the GRADE approach are summarized in online supplementary Tables 4 and 5 .

\section{Probiotics in VLBW Infants}

Effects of Probiotics on Severe NEC (Stage II-III)

Evidence from RCTs. Twenty-nine trials [19-47] reported data on NEC (stage II-III) in VLBW infants. The administration of probiotics significantly reduced the in- cidence of severe NEC (RR 0.57, 95\% CI 0.47-0.70, $p<$ 00001; Fig. 1). There was no evidence of significant publication bias by inspection of the funnel plot and formal statistical tests (Egger test, $p=0.072$; Begg test, $p=0.183$; online suppl. Fig. 2).

Evidence from Observational Studies. Fourteen studies [48-61] reported on severe stage II-III NEC. The administration of probiotics significantly reduced the incidence of severe stage II-III NEC in VLBW infants (RR 0.51, 95\% CI $0.37-0.70, p<0001$; Fig. 2). There was no evidence of significant publication bias by inspection of the funnel plot and formal statistical tests (Egger test, $p=0.616$; Begg test, $p=0.669$; online suppl. Fig. 2).

\section{Effects of Probiotics on Late-Onset Sepsis}

Evidence from RCTs. Twenty-eight trials [19-26, 28$35,37-47]$ reported on late-onset sepsis. The administration of probiotics reduced the rate of sepsis in the pooled effect by $12 \%$ (typical RR $0.88,95 \%$ CI $0.80-0.97, p=0.01$; Fig. 3). There was no evidence of significant publication 


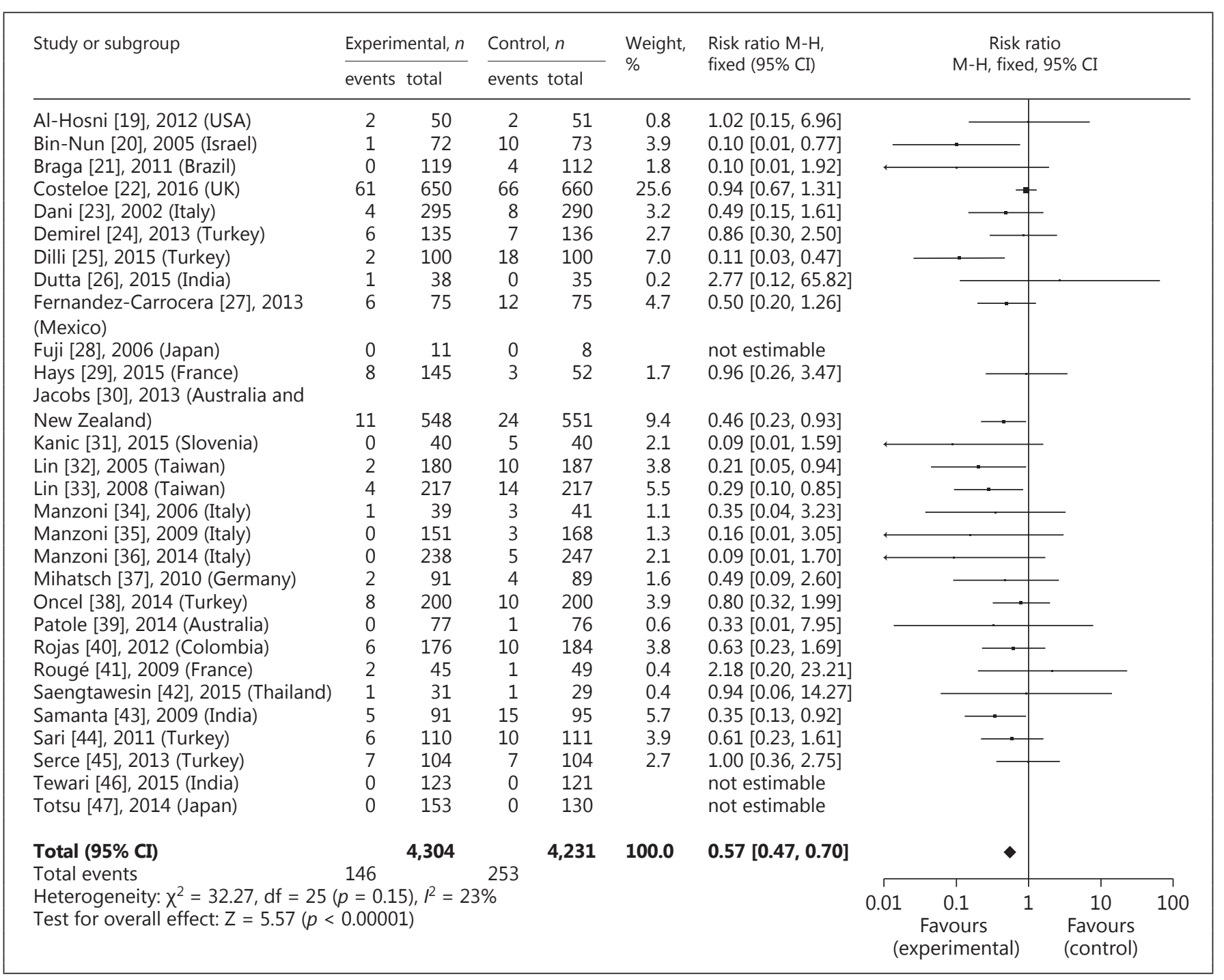

Fig. 1. Effects of probiotics on severe NEC (stage II-III) in RCT studies.

bias by inspection of the funnel plot and formal statistical tests (Egger test, $p=0.242$; Begg test, $p=0.149$; online suppl. Fig. 3).

Evidence from Observational Studies. Eight studies [48, 51-54, 58-60] reported on late-onset sepsis. The administration of probiotics reduced the incidence of sepsis in VLBW infants by $19 \%$ (RR $0.81,95 \%$ CI $0.69-0.96, p=$ 0.01 ; Fig. 4). There was no evidence of significant publication bias by inspection of the funnel plot and formal statistical tests (Egger test, $p=0.011$; Begg test, $p=0.108$; online suppl. Fig. 3).
Effects of Probiotics on Mortality

Evidence from RCTs. Twenty-seven trials [19-27, 29$39,41-47]$ reported on mortality. The administration of probiotics significantly reduced the rate of mortality in the VLBW infants (typical RR 0.77, 95\% CI 0.65-0.92, $p=0.003$; Fig. 5). Visual interpretation of this funnel plot showed some asymmetry, and the formal statistical tests showed significant publication bias (Egger test, $p=0.012$; Begg test, $p=0.002$; online suppl. Fig. 4).

Evidence from Observational Studies. Eleven studies [48-51, 53-56, 58-60] reported on mortality. The administration of probiotics significantly reduced the incidence of mortality in VLBW infants (RR 0.71, 95\% CI 0.62-0.81, 


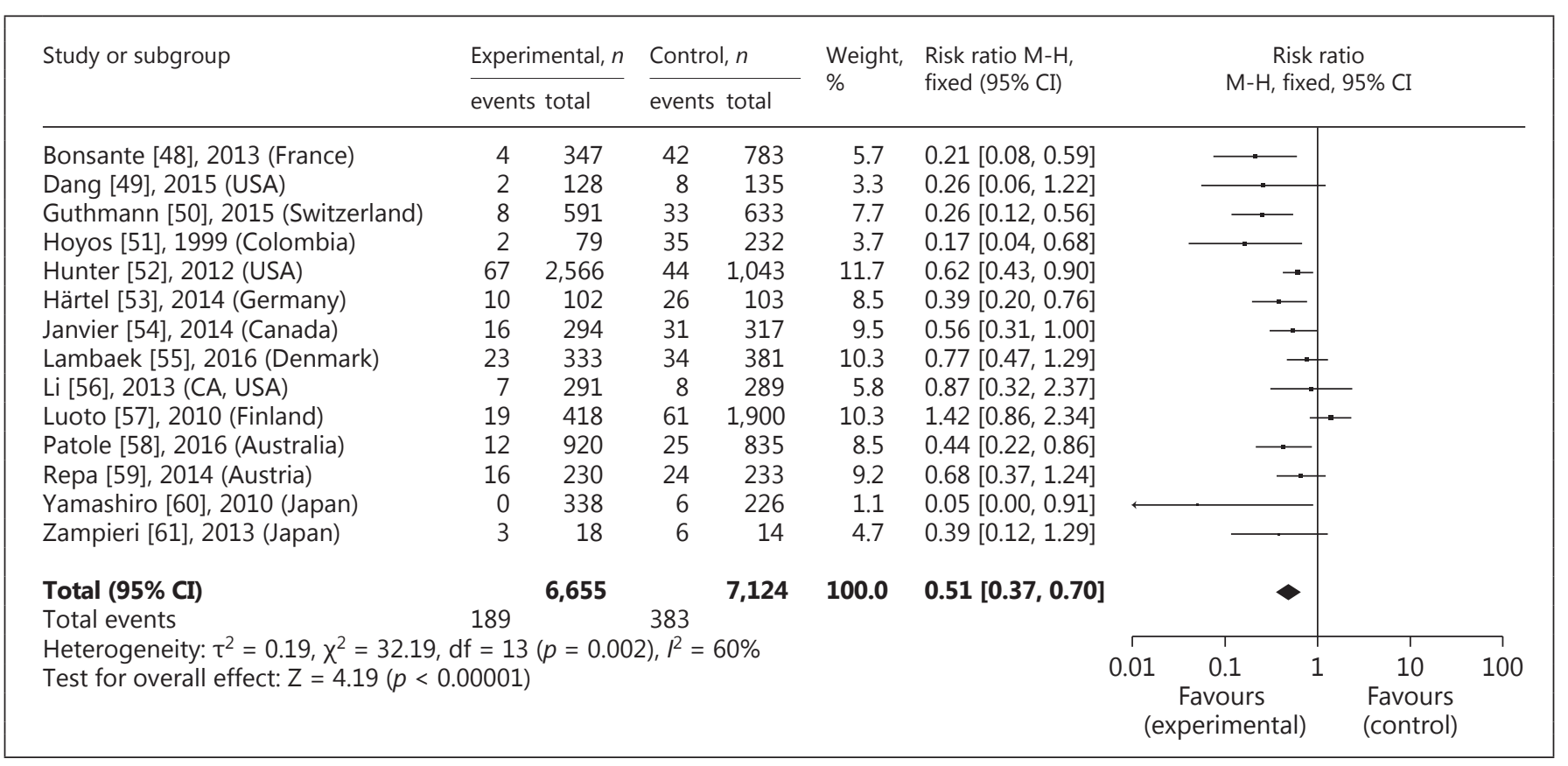

Fig. 2. Effects of probiotics on severe NEC (stage II-III) in observational studies.

$p<0.00001$; Fig. 6). There was no evidence of significant publication bias by inspection of the funnel plot and formal statistical tests (Egger test, $p=0.119$; Begg test, $p=0.082$; online suppl. Fig. 4).

\section{Probiotics in ELBW Infants}

Evidence from RCTs and Observational Studies

Three RCTs $[19,22,30]$ studied the effect of probiotics among ELBW infants. The study showed no statistically significant decrease of severe stage II-III NEC (typical RR $0.93,95 \%$ CI $0.67-1.27 ; p=0.64$, sepsis (typical RR 0.98, $95 \%$ CI $0.80-1.22 ; p=0.88$ ), or mortality (typical RR 0.89 , $95 \%$ CI 0.62-1.26; $p=0.50$; online suppl. Fig. 5).

Two observational studies $[52,54]$ involving a total of 518 infants (177 probiotic group and 341 control group), showed a reduction in the risk of NEC; however, this was not statistically significant (RR 0.37 ; $95 \%$ CI $0.10-1.38$; $p=0.14$ ). The study showed no statistically significant decrease of sepsis (typical RR 0.83, 95\% CI 0.62-1.11; $p=$ 0.21 ), or mortality (typical RR $0.58,95 \%$ CI $0.32-1.04$; $p=0.07$; online suppl. Fig. 5).

\section{Effect of Different Species of Probiotics}

Severe NEC: Species of Probiotics

Evidence from RCTs. The administration of Lactobacillus GG [23, 34-36] and Bifidobacterium lactis [25, 37, 29] species alone significantly reduced the incidence of severe stage II-III NEC (RR 0.31, 95\% CI 0.12-0.76, and RR $0.24,95 \%$ CI $0.10-0.58$, respectively). The pooled effect of the included trials that utilized L. reuteri $[38,40]$, B. breve $[22,39]$ and Saccharomyces boulardii alone [24, $45,62]$ showed a lack of significant reduction of severe NEC stage II-III (RR 0.71, 95\% CI 0.37-1.39, and RR 0.92 , 95\% CI $0.67-1.28$; RR 0.82, 95\% CI 0.44-1.50, respectively). Subgroup analysis identified that mixtures of 2 types and mixtures of more than 2 types of probiotics were most beneficial in reducing the risk of NEC (RR = $0.34 ; 95 \%$ CI $0.17-0.67, p=0.002$, and $\mathrm{RR}=0.40$; 95\% CI $0.26-0.64, p<0.0001$, respectively; online suppl. Fig. 6).

Evidence from Observational Studies. The administration of $L$. reuteri showed a significant reduction of the risk rate of NEC (RR 0.17, 95\% CI 0.04-0.68) [52]. There was no significant difference in NEC risk between the probiotic group and the control group for the infants receiving B. breve or Lactobacillus GG ( $\mathrm{RR}=0.27$; 95\% CI $0.02-$ $3.39, p=0.31$, and $\mathrm{RR}=0.58$; $95 \%$ CI $0.08-4.02, p=0.58$, respectively). Analysing the different strains, the use of a 


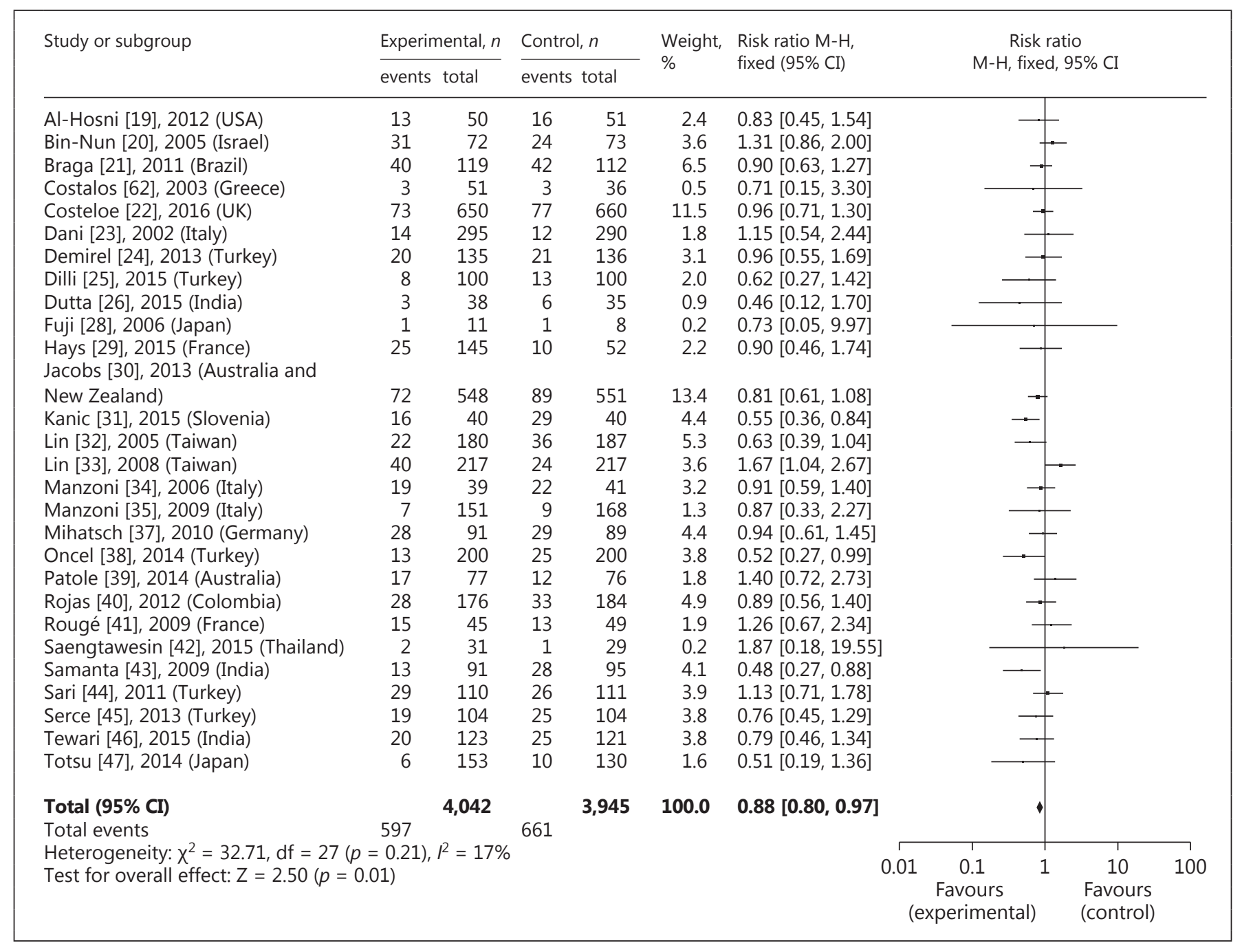

Fig. 3. Effects of probiotics on late-onset sepsis in RCT studies.

2-probiotic combination (L. acidophilus with B. infantis) proved to be statistically significant in reducing NEC when compared to other probiotic combinations (RR $0.49,95 \%$ CI 0.33-0.73, $p=0.0004$; online suppl. Fig. 6).

Late-Onset Sepsis: Species of Probiotics

Evidence from RCTs. The administration of single-strain L. reuteri, Lactobacillus GG, B. lactis, B. breve, S. boulardii alone or a mixture of 2 types of probiotics or more did not reduce the incidence of culture-proven sepsis significantly (RR 0.73, 95\% CI 0.50-1.05, RR 0.97, 95\% CI 0.67-1.40, RR 0.86, 95\% CI 0.61-1.22, RR 1.02, 95\% CI 0.78-1.34, RR 0.85, 95\% CI 0.58-1.25, RR 0.99, 95\% CI 0.80-1.23, and RR 0.82, 95\% CI 0.66-1.02, respectively; online suppl. Fig. 7).
Evidence from Observational Studies. Two observational studies $[58,60]$ using $B$. breve alone showed a statistically significant reduction in the risk of sepsis ( $\mathrm{RR}=0.66,95 \%$ CI $0.55-0.81, p<0.0001)$. The administration of single-strain L. reuteri, Lactobacillus GG or a mixture of 2 types of probiotics did not reduce the incidence of culture-proven sepsis (RR 0.77, 95\% CI 0.501.20, RR 0.64, 95\% CI 0.46-0.90, and RR 0.95, 95\% CI $0.77-1.17$, respectively; online suppl. Fig. 7).

\section{Mortality: Species of Probiotics}

Evidence from RCTs. The administration of singlestrain L. reuteri, Lactobacillus GG, B. lactis, B. breve or $S$. boulardii alone did not reduce mortality (RR 0.75, 95\% CI
16

Neonatology 2017;112:9-23 DOI: $10.1159 / 000454668$
Dermyshi/Wang/Yan/Hong/Qiu/Gong/ Zhang 


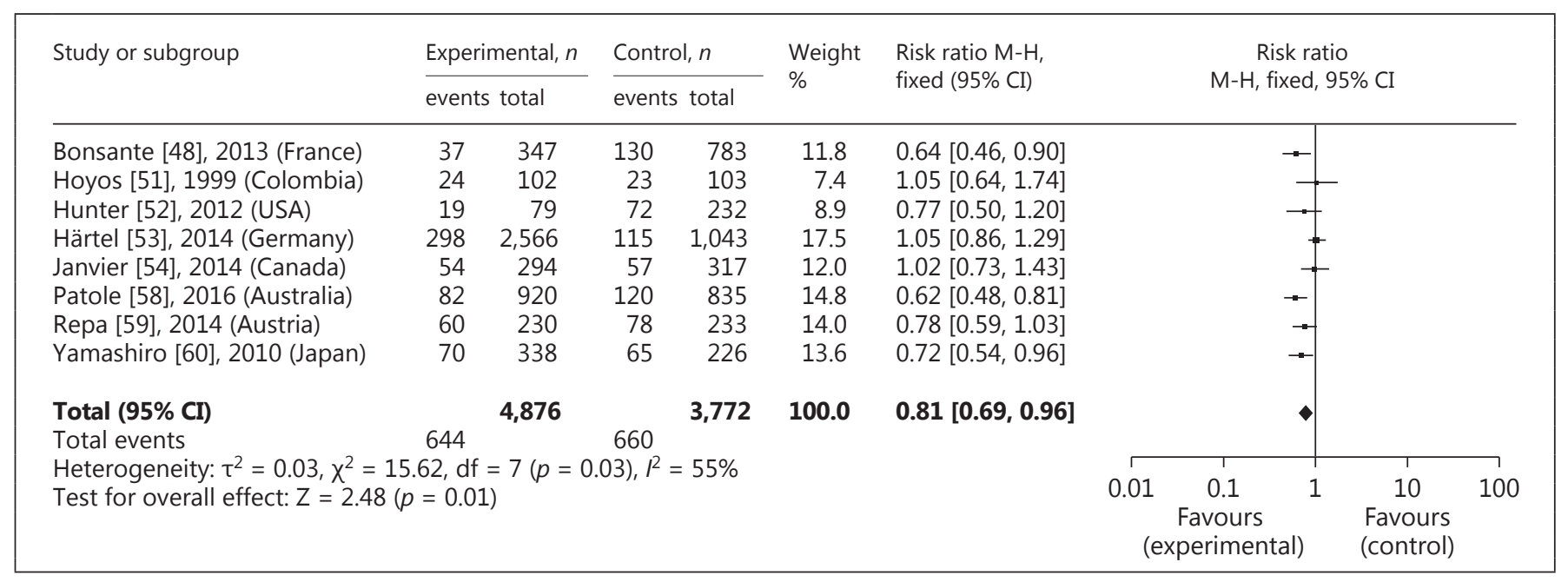

Fig. 4. Effects of probiotics on late-onset sepsis in observational studies.

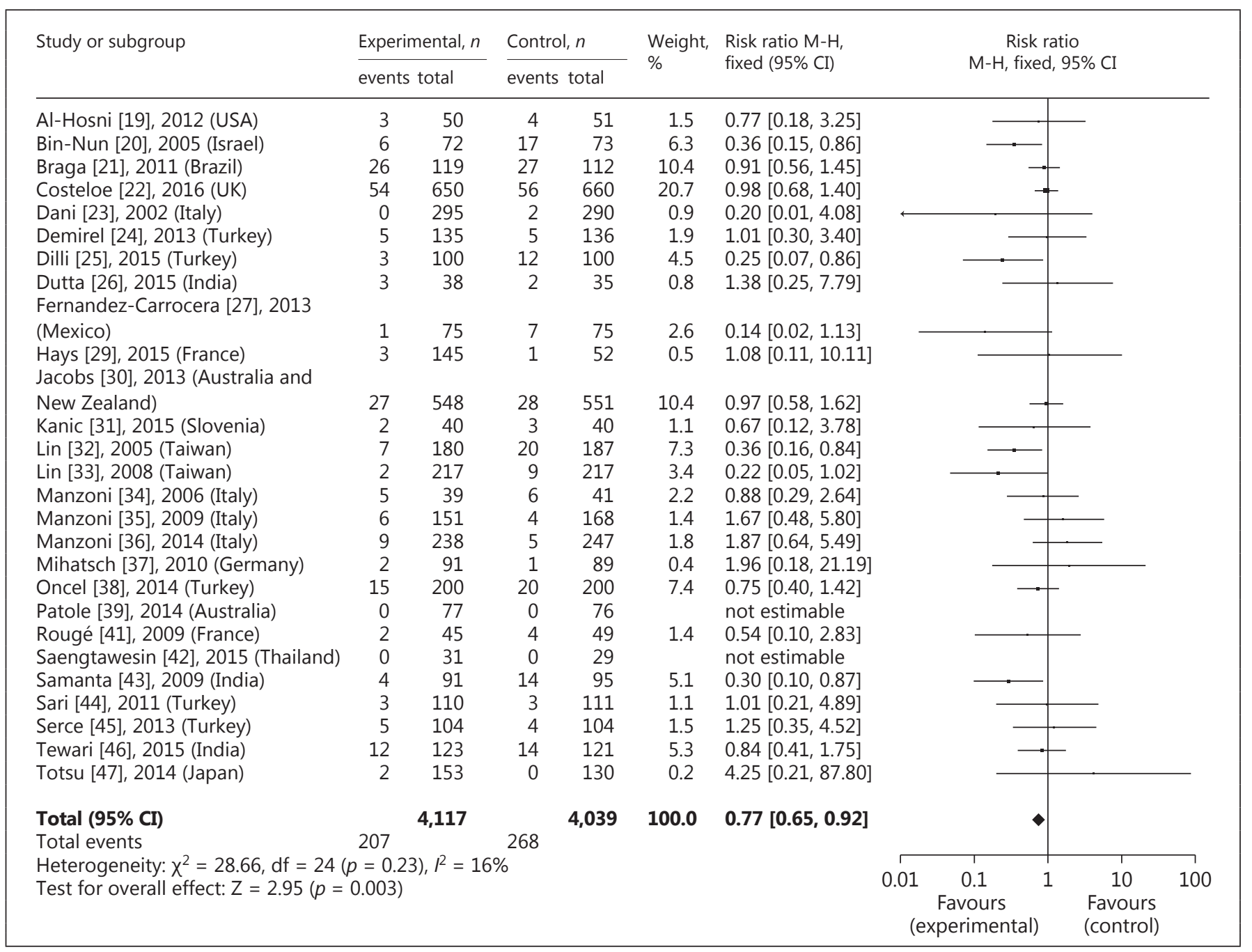

Fig. 5. Effects of probiotics on mortality in RCT studies. 


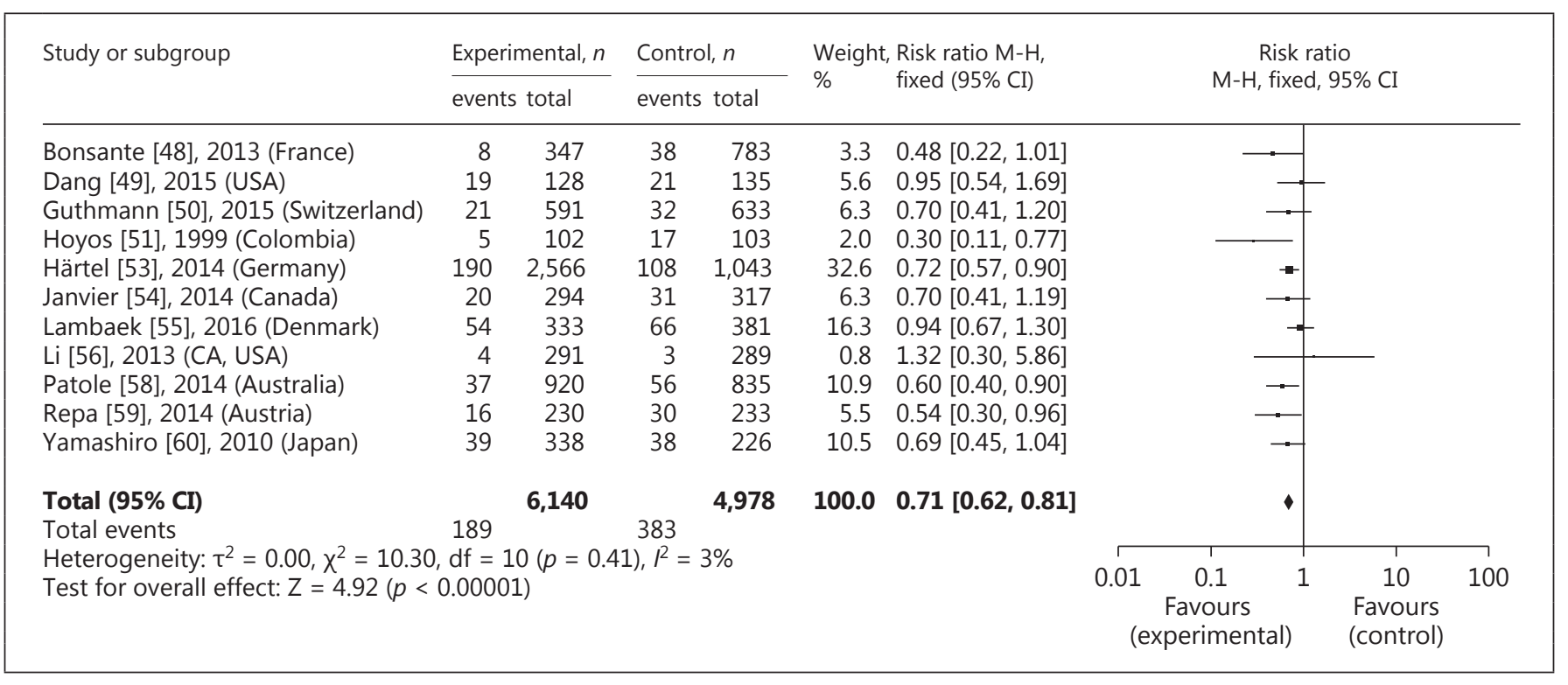

Fig. 6. Effects of probiotics on mortality in observational studies.

0.40-1.42, RR 1.16, 95\% CI 0.62-2.16, RR 0.54, 95\% CI $0.08-3.78$, RR 0.98 , 95\% CI 0.68-1.40, and RR 1.12, 95\% CI 0.46-2.70, respectively). The administration of a mixture of probiotics significantly reduced the incidence of mortality (RR $0.61,95 \%$ CI $0.43-0.89, p=0.009$; online suppl. Fig. 8).

Evidence from Observational Studies. The administration of $B$. breve alone or a mixture of probiotics significantly reduced the incidence of mortality (RR $0.64,95 \%$ CI $0.48-0.86, p=0.003$, and RR $0.64,95 \%$ CI $0.50-0.83$, $p=0.0006$, respectively; online suppl. Fig. 8).

\section{Discussion}

Over the last few years probiotics have been one of the most studied interventions in neonatal medicine. To some, probiotics appear to be the miracle cure of this century due to the fact that their use has been suggested to prevent severe NEC, late-onset sepsis, and to decrease mortality in preterm infants. The evidence for the efficacy and safety probiotics has been substantial compared to other innovative interventions such as surfactant, hypothermia, and room air resuscitation. However, is it really appropriate to say that this is the "golden age" of probiotics in preterm infants? Our systemic review and meta-analysis on all types of studies summarizes the evidence on probiotics efficacy in preterm infants weighing
$<1,500 \mathrm{~g}$. Thirty randomized trials with more than 8,000 preterm infants and 14 observational studies with more than 13,000 preterm infants were included. This study readily illustrates 3 significant findings.

The first finding is that there is a very sizeable volume of RCTs and observational studies on probiotics. Almost unanimously, they claim that the use of probiotics in VLBW infants is safe and feasible. This meta-analysis of RCT and observational studies found that the use of probiotics in premature infants was associated with a statistically significant decreased incidence of NEC, late-onset sepsis, and mortality. The effect sizes and CIs were also very similar in RCTs and observational studies, with an almost 50\% reduction in NEC and 25\% reduction in mortality. These results are consistent with the most recent Cochrane review and previous reviews that reported a $59 \%$ reduction in the risk of NEC and a 34\% reduction in all-cause mortality [9]. Our study demonstrated that probiotic supplementation also reduced the risk of lateonset sepsis in preterm infants. These results are in contrast to those of the latest meta-analyses that did not find a statistically significant benefit of probiotic supplementation in reducing late-onset sepsis in preterm infants $[9$, 64]. The most likely reason for the difference between our meta-analysis and the previous ones is the sample size.

The second finding brought out by the extremely high proportion of studies is that there is still insufficient clin-
18

Neonatology 2017;112:9-23 DOI: $10.1159 / 000454668$
Dermyshi/Wang/Yan/Hong/Qiu/Gong/ Zhang 
ical trial data available on which to consider the merits of both the safety and efficacy of providing probiotics to ELBW infants. Only 3 RCT studies $[19,22,30]$ and 2 observational studies $[52,54]$ specifically studied ELBW infants $<1,000 \mathrm{~g}$. Our meta-analysis showed a trend towards a benefit in the reduction of NEC, sepsis, and mortality; however, this was not statistically significant. Even though our study included a larger number of RCT and observational studies, it did not reach the sample size needed to detect a statistically significant benefit for probiotic use in ELBW infants. Therefore, the use of probiotics in extremely premature infants needs further investigation, especially considering the limited data from both the RCTs and the observational studies. Future studies using scrupulous analysis will be able to gradually uncover the true value of probiotics and confirm its place in mainstream practice in ELBW infants.

The third finding that emerged from the extreme variety of probiotics used in neonatology was that the results are conflicting. In some studies the authors suggested that different effects were observed when different strains were used [20,32, 37]. Others found no difference at all or suggested different effects when infants were supplemented with a single or with combined strains $[10,29$, 50]. The evidence indicates that the functionality of a multistrain or multispecies probiotics could be more effective and more consistent than that of a monostrain probiotics $[65,66]$. Our study demonstrated that oral $L$. reuteri does not seem to affect the overall rates of NEC and/or mortality in preterm infants, even though 1 observational study demonstrated a significant effect in reducing the incidence of NEC using the same strain in lower doses [52]. However, the conflicting results of RCTs and observational studies on the effect of $L$. reuteri in the prevention of severe NEC underline the importance of independent study comparing different doses of the same probiotics. Lactobacillus GG significantly reduced NEC but did not reveal a significant effect on the rates of late-onset sepsis and mortality in VLBW infants [23, 34, 35]. The meta-analyses on observational studies demonstrated a significant effect in reducing the incidence of NEC using the same strain but indicated heterogeneity across the studied populations. It is important to remember that some cases of sepsis attributable to Lactobacillus species have been documented in high-risk patients on rare occasions, and recently Dani et al. [67] reported 2 further cases of sepsis in preterm supplemented with L. rhamnosus GG [67-70]. The meta-analysis of studies that used a mixture of 2 types of probiotics showed a statistically significant decreased incidence of NEC and mortality, but there was a lack of significant reduction of late-onset sepsis. Using the probiotic preparation Infloran (a mixture of L. acidophilus and B. infantis), 1 RCT trial demonstrated a reduction of NEC and mortality by approximately $80 \%$ [32]. Pooled analyses of 4 observational studies [50, $51,53,59]$ showed a statistically significant $51 \%$ reduction of NEC and $46 \%$ reduction of mortality. Recently, a large observational multicentre study conducted by Denkel et al. [71] demonstrated that the routine use of dualstrain probiotics (Infloran) in German neonatal wards significantly reduced the risk of NEC, overall mortality, and late-onset sepsis. These effects were even more pronounced in the subgroup analysis of preterm infants with birth weights below $1,000 \mathrm{~g}$. In the meta-analysis of RCTs where a combination of more than 2 probiotics was used, a statistically significant effect in preventing NEC and mortality was seen. Furthermore, the meta-analysis identified a trend towards a benefit in the reduction of sepsis; however, this was not statistically significant. Our results indicated that multistrain probiotics showed greater efficacy than single strains.

The included trials were highly variable in terms of birth weight, gestational age, timing, dose, type of probiotics, and feeding regimens. They had different protocols towards enteral feeds, but almost unanimously they commenced probiotics within the first week of life. Doses of individual probiotics varied, and were administered with human milk feeding [21, 34, 48, 58], formula feeding [62], or both in some studies [23-25, 27, 32, 33]. Probiotic supplementation in some studies was started as soon as minimal enteral feeding was commenced $[23,38,48]$, or within $48 \mathrm{~h}[24,47,50]$, or when infants had stable vital signs $[21,32,33,37,40]$. In other studies it was initiated as soon as the infants could tolerate enteral feeding $[62,63]$, or as soon as possible after randomization, whether or not enteral feeding had begun [22]. Repa et al. [59] observed a preventive effect against NEC in premature infants fed with breast milk in the first 2 weeks of life, but not in infants exclusively fed with formula, suggesting that the efficacy of probiotics is strongly influenced by feeding practices. In fact, the health benefits of breastfeeding have been recognized for a long time. However, the above observations are made to highlight the need for a greater quality of evidence in terms of the preparation, dosage, and timing of probiotic supplementation in VLBW infants.

\section{Comparison with Previous Studies}

Differences between the current meta-analysis and previous meta-analyses conducted in the last 2 years 
should be noted. The latest meta-analysis had an obvious lack of effect of probiotics in ELBW infants, on the prevention of sepsis, and on the use of a specific probiotic strain. A meta-analysis by Baucells et al. [72] included 9 studies pooling a total of 3,521 newborns and found that probiotics administration was associated with a reduced risk of NEC (RR 0.39 ; 95\% CI 0.26-0.57) and mortality (RR 0.70; 95\% C 0.52-0.93), with no difference to placebo regarding late-onset sepsis (RR 0.91; 95\% CI 0.781.06).

Lau et al. [64] evaluated the effects of probiotic supplementation on the prevention of NEC in preterm infants. The authors included 20 RCTs involving 5,982 preterm infants. The risk of NEC was reduced by $49.1 \%$ (RR 0.509 , $95 \%$ CI $0.385-0.672$ ) and overall mortality by $26.9 \%$ (RR $0.731,95 \%$ CI $0.57-0.926$ ) among infants receiving probiotics. A reduction in late-onset sepsis of 8.1\% (RR 0.919, 95\% CI 0.823-1.027) was also observed in infants receiving probiotics, but this was not statistically significant.

Olsen et al. [73] included 12 observational studies with 10,800 premature neonates $(5,144$ receiving prophylactic probiotics and 5,656 controls). The meta-analysis showed a significantly decreased incidence of NEC (RR 0.55, 95\% CI 0.39-0.78) and mortality (RR 0.72, 95\% CI 0.61-0.85). Sepsis did not differ significantly between the 2 groups (RR 0.86, 95\% CI 0.74-1.00). The authors concluded that probiotic supplementation reduces the risk of NEC and mortality in preterm infants.

Aceti et al. [74] analysed data from 6,605 infants (3,324 in the probiotic group and 3,281 in the control group). Probiotics prevented NEC in preterm infants (RR 0.47, 95\% CI 0.36-0.60), and a strain-specific sub-meta-analysis showed a significant effect for bifidobacteria (RR 0.24 , 95\% CI 0.10-0.54) and for probiotic mixtures (RR 0.39, 95\% CI 0.27-0.56).

Following these meta-analyses, several RCTs and observational studies investigating probiotics for the prevention of NEC, late-onset sepsis or mortality were published. We used rigorous methods to systematically identify both randomized and observational studies that reported data to inform the issue of using probiotics in infants $<1,500 \mathrm{~g}$ or $<34$ weeks gestational age. Our search strategy involved multiple bibliographic databases and we did not exclude specific study types, making this the largest systematic review on probiotics in preterm infants. The inclusion of observational and RCT data gave the full picture of using probiotics in preterm infants. In contrast with the previous meta-analyses, the current meta-analysis of RCTs and observational studies with similar effect sizes suggested that probiotic supplementation might reduce the risk of sepsis significantly. Furthermore, it showed a statistically significant decrease in the incidence of NEC and mortality in both the RCTs and the observational studies that used a mixture of 2 or more types of probiotics.

Our study has some limitations. First, we included only trials published in English; other languages, ongoing registered trials, and abstracts presented in conferences were not included. Second, the different preparations, dosing, and the limited data on the highest-risk population (ELBW infants) of RCTs and observational studies made our analysis of the evidence difficult. Third, the available evidence is not suited to providing specific answers to some questions, since many findings came from RCTs and observational studies of very low-quality evidence. Studies based on observational data encounter methodological problems that can compromise the validity and bias our results. The methodological issues among the reviewed observational studies are the handling of exposures that change over time and difficulty in controlling for all confounders [58]. Bonsante et al. [48] could not draw any conclusion about the overall safety of the probiotic in VLBW infants because of the lack of a prospective and systematic surveillance of the microbiologic tolerability. The reduced rates of NEC associated with the introduction of probiotics might have been linked to other changes in practice, such as less antenatal antibiotics, more breastfeeding, or emphasis on late cord clamping $[50,54]$. Other concomitant changes in the practice of airway and sepsis management occurred in the study period and may have influenced some of the outcomes and the survival data [59]. Furthermore, the funnel plots and the statistical tests (effects of probiotics on mortality in RCTs) indicated publication bias. To interpret the accumulated evidence, it is necessary to make a judgment about the validity or relevance of the combined evidence from the smaller studies compared with that from larger studies. Although several reasons for small study effects exist, the main concern is that published studies might represent a biased selection of all the studies that have been conducted. We have compared the fixed and random effects estimates of the probiotic on mortality and there is no evidence of a beneficial effect reported from smaller studies. This suggests that publication bias does not completely explain the asymmetry, since many of the beneficial effects reported from smaller studies were not significant. Plausible explanations for these results are that changes in the standard of care (less antenatal antibiotics or more breastfeeding) led to the apparent beneficial effects of probiotics. 


\section{Conclusions}

This large meta-analysis supports the hypotheses that probiotics potentially prevent severe NEC and late-onset sepsis, and reduce mortality in preterm infants. Combinations containing $L$. acidophilus together with $B$. infantis or more should be strongly considered, as the available evidence does not support the single-strain use of $L$. reuteri, B. breve or $S$. boulardii. Analysing the different strands, the use of a 2-probiotic combination (L. acidophilus with $B$. infantis) proved to be statistically significant in reducing NEC and mortality when compared to other probiotic combinations.

As the neonatology community moves forward today, it is necessary for the role of probiotics in ELBW infants to be defined. Considering the similar results in this metaanalysis and the limited data on the efficacy and safety of using probiotics in infants at high risk, well-designed observational studies that utilise the same features of RCTs, such as inclusion and exclusion criteria, may be the next practicable method to provide valuable information about the use of probiotics in ELBW infants.

We are living in the golden age of probiotics, but the question is whether or not we should use them in VLBW infants? The answer currently is that the debate is a very important one to have. Despite the attractiveness of probiotics use in VLBW infants, proponents must under- stand that the counterarguments are very relevant. The reasons not to use some types of probiotics and the difficulty in deriving dosage recommendations or probiotics combinations are as important for patients as the reasons in favour of using them. These doubts must never be ignored or dismissed. Facing up to and seeking to resolve this debate is the only way to ensure that patients are the ultimate winners.

\section{Author Contributions}

E. Dermyshi had full access to all of the data in the study and takes responsibility for its integrity and the accuracy of the data analysis. Study concept and design: E. Dermyshi, Y. Wang, and T. Zhang conceived and designed the study, collected and analysed the data, and wrote the article. Acquisition of data: E. Dermyshi, Y. Wang, C. Yan, W. Hong. Analysis and interpretation of data: E. Dermyshi, Y. Wang, T. Zhang, G. Qiu, X. Gong. Drafting of the manuscript: E. Dermyshi, T. Zhang. Critical revision of the manuscript for important intellectual content: E. Dermyshi, Y. Wang, C. Yan, G. Qiu, X. Gong, T. Zhang. All authors approved the final version of the manuscript. Statistical analysis: E. Dermyshi. Administrative, technical, or material support: X. Gong. Study supervision: T. Zhang. Funding: none. Role of the sponsor: none.

\section{Disclosure Statement}

The authors have no conflicts of interest to disclose.

\section{References}

1 Berrington JE, Hearn RI, Bythell M, Wright C, Embleton ND: Deaths in preterm infants: changing pathology over 2 decades. J Pediatr 2012;160:49-53.

2 Lee JS, Polin RA: Treatment and prevention of necrotizing enterocolitis. Semin Neonatol 2003;8:449-459.

3 Neu J: Necrotizing enterocolitis: the mystery goes on. Neonatology 2014;106:289-295.

4 Dai D, Walker WA: Role of bacterial colonization in neonatal necro-tizing enterocolitis and its prevention. Zhonghua Min Guo Xiao Er Ke Yi Xue Hui Za Zhi 1998;39:357-365.

5 Neu J: Neonatal necrotizing enterocolitis: an update. Acta Paediatr 2005;94:100-105.

6 Lin HC, Hsu CH, Chen HL, Chung MY, Hsu JF, Lien RI, et al: Oral probiotics prevent necrotizing enterocolitis in very low birth weight preterm infants: a multicenter, randomized, controlled trial. Pediatrics 2008;122:693-700.

7 Mshvildadze M, Neu J, Mai V: Intestinal microbiota development in the premature neonate: establishment of a lasting commensal relationship? Nutr Rev 2008;66:658-663.
8 Cotton CM, Taylor S, Stoll B, Goldberg RN, Hansen NI, Sánchez PJ, et al: Net-work, prolonged duration of initial empirical antibiotic treatments associated with increased rates of necrotizing enterocolitis and death for extremely low birth weight infants. Pediatrics 2009; 123:58-66.

9 AlFaleh K, Anabrees J: Probiotics for prevention of necrotizing enterocolitis in preterm infants. Cochrane Database Syst Rev 2014; 4:CD005496.

10 Deshpande G, Rao S, Patole S, Bulsara M: Updated meta-analysis of probiotics for preventing necrotizing enterocolitis in preterm neonates. Pediatrics 2010;125:921-930.

11 Olsen R, Greisen G, Schroder M, Brok J: Prophylactic probiotics for preterm infants: a systematic review and meta-analysis of observational studies. Neonatology 2016;109:105112.

12 Cameron C, Fireman B, Hutton B, Clifford T, Coyle D, Wells G, et al: Network meta-analysis incorporating randomized controlled trials and nonrandomized comparative cohort studies for assessing the safety and effectiveness of medical treatments: challenges and opportunities. Syst Rev 2015;4:147.

13 Liberati A, Altman DG, Tetzlaff J, Mulrow C, Gøtzsche PC, Ioannidis JP, et al: The PRISMA statement for reporting systematic reviews and meta-analyses of studies that evaluate health care interventions: explanation and elaboration. J Clin Epidemiol 2009;62:e1-e34.

14 Stroup DF, Berlin JA, Morton SC, Olkin I, Williamson GD, Rennie D, et al: Meta-analysis of observational studies in epidemiology: a proposal for reporting. Meta-analysis Of Observational Studies in Epidemiology (MOOSE) group. JAMA 2000;283:2008-2012.

15 Higgins J, Green S (eds): Cochrane Handbook for Systematic Reviews of Interventions, version 5.1.0 (updated March 2011). The Cochrane Collaboration, 2011. www.handbook. cochrane.org.

16 Higgins JP, Altman DG, Gotzsche PC, Jüni P, Moher D, Oxman AD, et al: The Cochrane Collaboration's tool for assessing risk of bias in randomised trials. BMJ 2011;343:d5928. 
17 Guyatt G, Oxman AD, Akl EA, Kunz R, Vist G, Brozek J, et al: GRADE guidelines: 1. Introduction - GRADE evidence profiles and summary of findings tables. J Clin Epidemiol 2011;64:383-394.

18 Wells GA, Shea B, O'Connel D, Peterson J, Welch V, Losos M, et al: The Newcastle-Ottawa Scale (NOS) for assessing the quality of nonrandomised studies in meta-analyses 2013. Ottawa, University of Ottawa, 2013. http://www.ohri.ca/programs/clinical_epidemiology/oxford.asp.

19 Al-Hosni M, Duenas M, Hawk M, Stewart LA Borghese RA, Cahoon M, et al: Probioticssupplemented feeding in extremely low-birthweight infants. J Perinatol 2012;32:253-259.

20 Bin-Nun A, Bromiker R, Wilschanski M, Kaplan M, Rudensky B, Caplan M, et al: Oral probiotics prevent necrotizing enterocolitis in very low birth weight neonates. J Pediatr 2005; 147:192-196.

21 Braga TD, da Silva GAP, de Lira PI, de Carvalho LM: Efficacy of Bifidobacterium breve and Lactobacillus casei oral supplementation on necrotizing enterocolitis in very-lowbirth-weight preterm infants: a double blind, randomized, controlled trial. Am J Clin Nutr 2011;93:81-86.

22 Costeloe K, Hardy P, Juszczak E, Wilks M, Millar MR, et al: Bifidobacterium breve BBG-001 in very preterm infants: a randomised controlled phase 3 trial. Lancet 2016;387:649-660.

23 Dani C, Biadaioli R, Bertini G, Martelli E, Rubaltelli FF: Probiotics feeding in prevention of urinary tract infection, bacterial sepsis and necrotizing enterocolitis in preterm infants. Biol Neonate 2002;82:103-108.

24 Demirel G, Erdeve O, Celik IH, Dilmen U: Saccharomyces boulardii for prevention of necrotizing enterocolitis in preterm infants: a randomized, controlled study. Acta Paediatr 2013; 102:560-565.

25 Dilli D, Aydin B, Fettah ND, Özyazıcı E, Beken S, Zenciroğlu A, et al: The ProPre-Save study: effects of probiotics and prebiotics alone or combined on necrotizing enterocolitis in very low birth weight infants. J Pediatr 2015; 166:545-551.e1.

26 Dutta S, Ray P, Narang A: Comparison of stool colonization in premature infants by three dose regimes of a probiotic combination: a randomized controlled trial. Am J Perinatol 2015;32:733-740.

27 Fernández-Carrocera LA, Solis-Herrera A, Cabanillas-Ayón M, Gallardo-Sarmiento RB, García-Pérez CS, Montaño-Rodríguez R, et al: Double-blind, randomised clinical assay to evaluate the efficacy of probiotics in preterm newborns weighing less than $1500 \mathrm{~g}$ in the prevention of necrotizing enterocolitis. Arch Dis Child Fetal Neonatal Ed 2013;98:F5-F9.

28 Fujii T, Ohtsuka Y, Lee T, Kudo T, Shoji H, Sato $\mathrm{H}$, et al: Bifidobacterium breve enhances transforming growth factor A1 signaling by regulating Smad7 expression in preterm infants. J Pediatr Gastroenterol Nutr 2006;43: $83-88$.
29 Hays S, Jacquot A, Gauthier H, Kempf C, Beissel A, Pidoux O, et al: Probiotics and growth in preterm infants: a randomized controlled trial, PREMAPRO study. Clin Nutr 2016;35:802-811.

30 Jacobs SE, Tobin JM, Opie GF, Donath S, Tabrizi SN, Pirotta M, et al: Probiotic effects on late-onset sepsis in very preterm infants: a randomized controlled trial. Pediatrics 2013; 132:1055-1062.

31 Kanic Z, Micetic Turk D, Burja S, Kanic V, Dinevski $D$, et al: Influence of a combination of probiotics on bacterial infections in very low birthweight newborns. Wien Klin Wochenschr 2015;127(suppl 5):S210-S215.

32 Lin HC, Su BH, Chen AC, Lin TW, Tsai CH, Yeh TF, et al: Oral probiotics reduce the incidence and severity of necrotizing enterocolitis in very low birth weight infants. Pediatrics 2005;115:1-4

33 Lin HC, Hsu CH, Chen HL, Chung MY, Hsu JF, Lien R, et al: Oral probiotics prevent necrotizing enterocolitis in very low birth weight preterm infants: a multicenter, randomized, controlled trial. Pediatrics 2008;122:693-700.

34 Manzoni P, Mostert M, Leonessa ML, Priolo C, Farina D, Monetti C, et al: Oral supplementation with Lactobacillus casei subspecies rhamnosus prevents enteric colonization by Candida species in preterm neonates: a randomized study. Clin Infect Dis 2006;42:17351742.

35 Manzoni P, Rinaldi M, Cattani S, Pugni L, Romeo MG, Messner H: Bovine lactoferrin supplementation for prevention of late-onset sepsis in very low-birth-weight neonates. JAMA 2009;302:1421-1428.

36 Manzoni P, Meyerb M, Stolfi I, Rinaldi M, Cattani S, Pugni L, et al: Bovine lactoferrin supplementation for prevention of necrotizing enterocolitis in very-low-birth-weight neonates: a randomized clinical trial. Early Hum Dev 2014;90(suppl 1):S60-S65.

37 Mihatsch WA, Vossbeck S, Eikmanns B, Hoegel J, Pohlandt F: Effect of Bifidobacterium lactis on the incidence of nosocomial infections in very-low-birth-weight infants: a randomized controlled trial. Neonatology 2010; 98:156-163.

38 Oncel MY, Sari FN, Arayici S, Guzoglu N, Erdeve O, Uras N, et al: Lactobacillus reuteri for the prevention of necrotising enterocolitis in very low birth weight infants: a randomised controlled trial. Arch Dis Child Fetal Neonatal Ed 2014;99:F110-F115.

39 Patole S, Keil AD, Chang A, Nathan E, Doherty D, Simmer K, et al: Effect of Bifidobacterium breve $\mathrm{M}-16 \mathrm{~V}$ supplementation on fecal bifidobacteria in preterm neonates - a randomised double blind placebo controlled trial. PLoS One 2014;9:e89511.

40 Rojas MA, Lozano JM, Rojas MX, Rodriguez VA, Rondon MA, Bastidas JA, et al: Prophylactic probiotics to prevent death and nosocomial infection in preterm infants. Pediatrics 2012;130:e1113-e1120.
41 Rougé C, Piloquet H, Butel MJ, Berger B, Rochat F, Ferraris L, et al: Oral supplementation with probiotics in very-low-birth-weight preterm infants: a randomized, double-blind, placebo-controlled trial. Am J Clin Nutr 2009; 89:1828-1835.

42 Saengtawesin V, Tangpolkaiwalsak R, Kanjanapattankul W: Effect of oral probiotics supplementation in the prevention of necrotizing enterocolitis among very low birth weight preterm infants. J Med Assoc Thai 2014;97:S20-S25.

43 Samanta M, Sarkar M, Ghosh P, Ghosh JK, Sinha MK, Chatterjee S: Prophylactic probiotics for prevention of necrotizing enterocolitis in very low birth weight newborns. J Trop Pediatr 2009;55:128-131.

44 Sari FN, Dizdar EA, Oguz S, Erdeve O, Uras N, Dilmen U: Oral probiotics: Lactobacillus sporogenes for prevention of necrotizing enterocolitis in very low-birth weight infants: a randomized, controlled trial. Eur J Clin Nutr 2011;65:434-439.

45 Serce O, Benzer D, Gursoy T, Karatekin G Ovali F: Efficacy of Saccharomyces boulardii on necrotizing enterocolitis or sepsis in very low birth weight infants: a randomised controlled trial. Early Hum Dev 2013;89:10331036.

46 Tewari VV, Dubey SK, Gupta G: Bacillus clausii for prevention of late-onset sepsis in preterm infants: a randomized controlled trial. J Trop Pediatr 2015;61:377-385.

47 Totsu S, Yamasaki C, Terahara M, Uchiyama A, Kusuda S, et al: Bifidobacterium and enteral feeding in preterm infants: cluster-randomized trial. Pediatr Int 2014;56:714-719.

48 Bonsante F, Iacobelli S, Gouyon JB: Routine probiotic use in very preterm infants: retrospective comparison of two cohorts. Am J Perinatol 2013;30:41-46.

49 Dang S, Shook L, Garlitz K, Hanna M, Desai $\mathrm{N}$ : Nutritional outcomes with implementation of probiotics in preterm infants. J Perinatol 2015;35:447-450.

50 Guthmann F, Arlettaz Mieth RP, Bucher HU, Bührer C: Short courses of dual-strain probiotics appear to be effective in reducing necrotising enterocolitis. Acta Paediatr 2015;255259 .

51 Hoyos AB: Reduced incidence of necrotizing enterocolitis associated with enteral administration of Lactobacillus acidophilus and Bifidobacterium infantis to neonates in an intensive care unit. Int J Infect Dis 1999;3:197-202.

52 Hunter C, Dimaguila MA, Gal P, Wimmer JE Jr, Ransom JL, Carlos RQ, et al: Effect of routine probiotic, Lactobacillus reuteri DSM 17938, use on rates of necrotizing enterocolitis in neonates with birthweight $<1,000$ grams: a sequential analysis. BMC Pediatr 2012;12:142.

53 Härtel C, Pagel J, Rupp J, Bendiks M, Guthmann F, Rieger-Fackeldey E, et al: Prophylactic use of Lactobacillus acidophilus/Bifidobacterium infantis probiotics and outcome in very low birth weight infants. J Pediatr 2014;165: 285-289.e1. 
54 Janvier A, Malo J, Barrington KJ: Cohort study of probiotics in a North American neonatal intensive care unit. J Pediatr 2014;164: 980-985.

55 Lambæk ID, Fonnest G, Gormsen M, Brok J, Greisen G: Routine probiotic prophylaxis for necrotizing enterocolitis in very preterm infants: a planned historically controlled study. Dan Med J 2016;63:A5203.

56 Li D, Rosito G, Slagle T: Probiotics for the prevention of necrotizing enterocolitis in neonates: an 8-year retrospective cohort study. J Clin Pharm Ther 2013;38:445-449.

57 Luoto R, Matomäki J, Isolauri E, Lehtonen L: Incidence of necrotizing enterocolitis in very low-birth-weight infants related to the use of Lactobacillus GG. Acta Paediatr 2010;99: 1135-1138.

58 Patole SK, Rao SC, Keil AD, Nathan EA, Doherty DA, Simmer KN: Benefits of Bifidobacterium breve $\mathrm{M}-16 \mathrm{~V}$ supplementation in preterm neonates - a retrospective cohort Study. PLoS One 2016;11:e0150775.

59 Repa A, Thanhaeuser M, Endress D, Weber M, Kreissl A, Binder C, et al: Probiotics (Lactobacillus acidophilus and Bifidobacterium bifidum) prevent NEC in VLBW infants fed breast milk but not formula. Pediatr Res 2015; 77:381-388.

60 Yamashiro Y, Nagata S: Beneficial microbes for premature infants, and children with malignancy undergoing chemotherapy. Benef Microbes 2010;1:357-365.
61 Zampieri N, Pietrobelli A, Biban P, Soffiati M, Dall'Agnola A, Camoglio FS: Lactobacillus paracasei subsp. paracasei F19 in Bell's stage 2 of necrotizing enterocolitis. Minerva Pediatr 2013;65:353-360.

62 Costalos C, Skouteri V, Gounaris A, Sevastiadou S, Triandafilidou A, Ekonomidou C, et al: Enteral feeding of premature infants with Saccharomyces boulardii. Early Hum Dev 2003;74:89-96.

63 Janvier A, Malo J, Barrington KJ: Cohort study of probiotics in a North American neonatal intensive care unit. J Pediatr 2014;164: 980-985.

64 Lau CS, Chamberlain RS: Probiotic administration can prevent necrotizing enterocolitis in preterm infants: a meta-analysis. J Pediatr Surg 2015;50:1405-1412.

65 Timmerman HM, Koning CJM, Mulder L, Rombouts FM, Beynen AC: Monostrain, multistrain and multispecies probiotics - a comparison of functionality and efficacy. Int J Food Microbiol 2004;96:219-233.

66 Chapman CM, Gibson GR, Rowland I: Health benefits of probiotics: are mixtures more effective than single strains? Eur J Nutr 2011;50: $1-17$.

67 Dani C, Coviello C, Corsini I, Arena F, Antonelli A, Rossolini GM: Lactobacillus sepsis and probiotic therapy in newborns: two new cases and literature review. AJP Rep 2016; 06:e25-e29.

68 Kunz AN, Noel JM, Fairchok MP: Two cases of Lactobacillus bacteremia during probiotic treatment of short gut syndrome. J Pediatr Gastroenterol Nutr 2004;38:457-458.
69 De Groote MA, Frank DN, Dowell E, Glode MP, Pace NR: Lactobacillus rhamnosus GG bacteremia associated with probiotic use in a child with short gut syndrome. Pediatr Infect Dis J 2005;24:278-280.

70 Land $\mathrm{MH}$, Rouster-Stevens K, Woods CR, Cannon ML, Cnota J, Shetty AK: Lactobacillus sepsis associated with probiotic therapy. Pediatrics 2005;115:178-181.

71 Denkel LA, Schwab F, Garten L, Geffers C, Gastmeier P, Piening B: Protective effect of dual-strain probiotics in preterm infants: a multi-center time series analysis. PLoS One 2016;11:e0158136.

72 Baucells BJ, Mercadal Hally M, Álvarez Sánchez AT, Figueras Aloy J: Probiotic associations in the prevention of necrotizing enterocolitis and the reduction of late-onset sepsis and neonatal mortality in preterm infants under 1500 g: a systematic review. An Pediatr (Barc) 2016;85:247-255

73 Olsen R, Greisen G, Schroder M, Brok J: Prophylactic probiotics for preterm infants: a systematic review and meta-analysis of observational studies. Neonatology 2016;109:105112

74 Aceti A, Gori D, Barone G, Callegari ML, Di Mauro A, Fantini MP, et al: Probiotics for prevention of necrotizing enterocolitis in preterm infants: systematic review and metaanalysis. Ital J Pediatr 2015;41:89. 